\title{
A Resource Scheduling Strategy for WSN with Communication Constrained
}

\author{
Kuan $\mathrm{Li}^{1, \mathrm{a}}$, Xiaoquan $\mathrm{Xu}^{2}$ \\ ${ }^{1}$ Institute of detection technology and smart sensing, Chongqing University of Posts and Telecommunications, Chongqing, China \\ ${ }^{2}$ Institute of detection technology and smart sensing, Chongqing University of Posts and Telecommunications, Chongqing, China
}

\begin{abstract}
Due to the loss of wireless communication link, the remote estimator in the wireless sensor network can only receive part of the observation information or can not be completely received, which reduces the accuracy of the state estimation. Focusing on the above problems, a strategy based on network resource scheduling is proposed to improve the impact of link loss on state estimation. The strategy considers the quantization process of sensor observations and the limited transmission bandwidth. The objective of optimization is to minimize the estimated error covariance and the expected energy consumption of the data packet. The data rate and the time slot are allocated to each communication link. The simulation results show that the optimal state estimation of the physical process can be obtained under a small transmission bandwidth and simple BPSK modulation, and the energy consumption of the transmitted data packet can be effectively reduced.
\end{abstract}

\section{Introduction}

Wireless sensor networks can greatly improve the ability to monitor and control the physical environment, the typical application is the environmental control[1], car networking and traffic control[2], inventory tracking[3] and military applications[4]. An important challenge in the wireless network is to improve the performance and reliability of the system under the constraints of network resources, including energy, transmission bandwidth, data rate, and so on.

Two important limitations of wireless communication includes: limited transmission bandwidth and information loss. In the field of networked state estimation studies, many studies assume that there is no quantification problems[5], but only consider the link packet loss problem. There are some studies, assuming that there is no link packet loss problem, but only consider the observation information and control information quantization process[6]. In the linear system, the minimized data rate is achieved in the packet loss network around the target state tracking control problem[7]. In the state of remote estimation of simple random linear system, due to quantization noise and communication link packet loss. You $\mathrm{K}$ researches coding and decoding methods[8].

This paper is a comprehensive consideration of wireless sensor network resource optimization,By establishing the covariance of the estimated error covariance and the energy of the packet transmission, through optimizing the target, the network resources are scheduled among the plurality of communication links, the transmission bandwidth, the link data rate and the transmission period are appropriately allocated,The estimation accuracy and the packet transmission energy are also considered while improving the estimation accuracy of the remote estimator for the physical process.The results of network resource optimization analysis show that the optimal state estimation of physical process can be obtained under the very small transmission bandwidth with simple BPSK modulation mode, and the energy of the transmission packet can be effectively reduced.

\section{System model and problem description}

\subsection{Dynamic physical processes and sensor observations}

The system structure contains two wireless communication links, two links between the independent transmission of data and sharing network resources.This paper discusses the general multi-input multi-output discrete-time linear system, Observation vector $s_{k}$ is divided into two parts $\left[s_{1, k}, s_{2, k}\right]$. The dynamic equation of the physical process is as follows:

$$
x_{k+1}=A x_{k}+\omega_{k}, k \geq 0
$$

Among them $x_{k} \in \mathbb{R}^{n}$ is the state variable of the physical process. The covariance matrix of physical process noise $\omega_{k}$ is $Q$. The observation equation for the sensor is as follows:

$\overline{\text { a Corresponding author: adamleek@ } 163 . c o m}$ 


$$
\left[s_{1, k}, s_{2, k}\right]^{T}=\left[C_{1}, C_{2}\right]^{T} x_{k}+\left[v_{1, k}, v_{2, k}\right]^{T}
$$

Among them $s_{1, k}, v_{1, k} \in \mathbb{R}^{m_{1}} \quad, \quad s_{2, k}, v_{2, k} \in \mathbb{R}^{m_{2}}$. Covariance matrix of observation noise $v_{1, k}$ and $v_{2, k}$ is $R_{11}$ and $R_{22}$.

\subsection{Observation information quantization process}

Observations $s_{1, k}$ and $s_{2, k}$ are quantified and encoded independently by two different sensors. And they are sent to the remote estimator by the communication channel. Assume that the transmitted packet contains an error detection code, so that the estimator can know whether the packet has an error when it receives the packet. When the observation packet is successfully received, the state estimation can be made; When the observation packet information is lost or there is an error, the observation information is discarded directly. For simplicity, each link is modeled as a single-hop network with no packet retransmission mechanism. If both signal quantization coding and packet loss are considered at the same time, then the estimator receives the signal as follows:

$$
y_{i, k}=\gamma_{i, k}\left(s_{i, k}+n_{i, k}\right)=\gamma_{i, k}\left(C_{i} x_{k}+v_{i, k}+n_{i, k}\right)
$$

variable $\gamma_{i, k}$ indicates whether the estimator receives the observations $y_{i, k}$. Assume that $\gamma_{1, k}$ and $\gamma_{2, k}$ are both independent distribution of Bernoulli random variables. $n_{i, k}$ is the observed quantization noise, assume that the quantization noise is a zero mean independent distribution of Gaussian noise. The covariance matrix is $R_{N_{i}}, \quad n_{i, k}$ is independent of the source signal. Definition $\bar{v}_{i, k}=v_{i, k}+n_{i, k}, \quad i=1,2$. As the equivalent noise of the observed information quantization, it is also zero mean Gaussian noise, covariance is $\bar{R}_{i}=R_{i i}+R_{N_{i}}$.

Suppose observation noise $v_{i, k}$ has the following conditional probability density distribution:

$$
p\left(v_{i, k} \mid \gamma_{i, k}\right) \sim \begin{cases}\mathrm{N}\left(0, R_{i i}\right), & \gamma_{i, k}=1 \\ \mathrm{~N}\left(0, \sigma_{i}^{2} I\right), & \gamma_{i, k}=0\end{cases}
$$

when $\sigma_{i}^{2} \rightarrow \infty$, indicates the observation information $y_{i, k}$ lost. According to the Kalman filter principle, we can get the following equation:

$$
\begin{aligned}
& P_{k+1}=A P_{k} A^{\prime}+Q-\gamma_{1, k} \gamma_{2, k} A P_{k} C^{\prime}\left(C P_{k} C^{\prime}+\bar{R}\right)^{-1} C P_{k} A^{\prime} \\
& -\gamma_{1, k}\left(1-\gamma_{2, k}\right) A P_{k} C_{1}^{\prime}\left(C_{1} P_{k} C_{1}^{\prime}+\bar{R}_{1}\right)^{-1} C_{1} P_{k} A^{\prime} \\
& -\left(1-\gamma_{1, k}\right) \gamma_{2, k} A P_{k} C_{2}^{\prime}\left(C_{2} P_{k} C_{2}^{\prime}+\bar{R}_{2}\right)^{-1} C_{2} P_{k} A^{\prime}
\end{aligned}
$$

\subsection{Forward fading communication channel}

Assume that the forward fading channel between the sensor and the estimator is unstable, $\gamma_{i, k}=1$ indicates that the packet was transmitted successfully, $\gamma_{i, k}=0$ indicates that packet transmission is failed. Set the probability of packet loss is $p, p \in[0,1]$. According to the previous description, $\mathrm{P}\left(\gamma_{i, k}=1\right)=1-p$. Assume that $p_{b}$ is the bit error rate for sending a bit of data, then send a packet with $n$ bits of data packet loss probability is

$$
p=1-\left(1-p_{b}\right)^{n}
$$

In a wireless communication channel, $p_{b}$ is related with the transmission energy $E_{b}$ of each bits. When $E_{b}$ increases, $p_{b}$ reduces. For different communication channels and different digital modulation modes, the calculation of $p_{b}$ is different. For example, In the BPSK modulation mode, the calculation of $p_{b}$ is as follows:

$$
p_{b}=Q\left(\sqrt{\frac{2 E_{b}}{N_{0}}}\right)
$$

Among them, $N_{0} / 2$ is the noise energy spectral density, the function $Q$ is:

$$
Q(x)=(1 / \sqrt{2 \pi}) \int_{x}^{\infty} e^{-t^{2} / 2} d t=(1 / 2) \operatorname{erfc}(x / \sqrt{2})
$$

\subsection{Objective function}

According to the foregoing description, the energy consumption of transmitting one bit of data in the channel is

$$
E_{b}=N_{0} \times\left(\operatorname{erfc}^{-1}\left(2 p_{b}\right)\right)^{2}
$$

Define the energy required to transfer a packet consisting of $B$ bits of data is as follows:

$$
\operatorname{energy}(k)=B_{k} \times E_{b}
$$

The goal of this paper is to design an optimal network resource scheduling method to minimize the covariance of the error and the energy required to transmit the packet. This optimization problem can be described as an optimization problem for infinite time domain:

$$
J=\limsup _{T \rightarrow \infty} \frac{1}{T} \sum_{k=0}^{T-1} \mathrm{E}\left[\beta \operatorname{Tr} P_{k}+(1-\beta) \sum_{i=1}^{2} \text { enery }_{i}(k)\right]
$$

\section{Network resource optimization}

Each link can be assigned to a portion of the period $T_{i}$ ( $i=1,2$ are two different links). Assume that the total transmission bandwidth is $W_{B}$. When the sensor uses phase shift keying modulation (BPSK or QPSK), 
assuming that the signals are transmitted independently, the probability of a packet reception is as follows when a packet of $B_{i}$ bits are transmitted in a Rayleigh fading channel [7] :

$$
\bar{\gamma}_{i, k}= \begin{cases}{\left[\frac{1}{2}\left(1+\sqrt{\frac{\Gamma_{i}}{\Gamma_{i}+1}}\right)\right]^{B_{i}}} & \text { BPSK } \\ \left(\sqrt{\frac{\Gamma_{i}}{\Gamma_{i}+2}}\right)^{B_{i} / 2} & \text { QPSK }\end{cases}
$$

When the sensor uses adaptive bit rate allocation technology (ad-RA), the probability of packet loss is :

$$
p_{\text {out }, i}\left(b_{i}, T_{i}\right)=1-\exp \left(\frac{-\left(2^{B_{i} /\left(W_{B} T_{i}\right)}-1\right)}{\Gamma_{i}}\right)
$$

The packet reception rate can be obtained :

$$
\bar{\gamma}_{i, k}=1-p_{\text {out }, i}\left(b_{i}, T_{i}\right), \quad i=1,2
$$

Therefore, the optimization method of network resource scheduling in this paper can be expressed as follows under BPSK / QPSK modulation mode:

$$
\left\{\begin{aligned}
\min & J \\
\text { s.t. } & T_{i} \geq 0 \\
& \sum_{i=1}^{3} T_{i} \leq T \\
\bar{P}_{k}= & g_{\lambda_{1} \lambda_{2}}\left(\bar{P}_{k}\right)
\end{aligned}\right.
$$

In ad-RA mode, the optimization method of network resource scheduling is expressed as follows:

$$
\left\{\begin{array}{cc}
\min & J \\
\text { s.t. } & T_{i} \geq 0, B_{i} \geq 0 \\
& \sum_{i=1}^{3} T_{i}=T \\
\bar{P}_{k}= & g_{\lambda_{1} \lambda_{2}}\left(\bar{P}_{k}\right)
\end{array}\right.
$$

\section{Analysis of network resource scheduling}

This section evaluates the performance of network resource scheduling.

Figure 1 is the relationship between the estimated error covariance and the packet loss probability with $\beta=1$. It can be found that in the absence of resource scheduling, if the link communication quality deteriorates, resulting in packet loss of the observed information, directly reduce the accuracy of the state estimation. At the same time, it can also be found under the BPSK modulation mode, the estimated error increases with the increasing of packet loss probability not changing so fast as the ad-RA modulation mode. It shows that the estimated error covariance is more stable and reliable under the BPSK modulation mode.

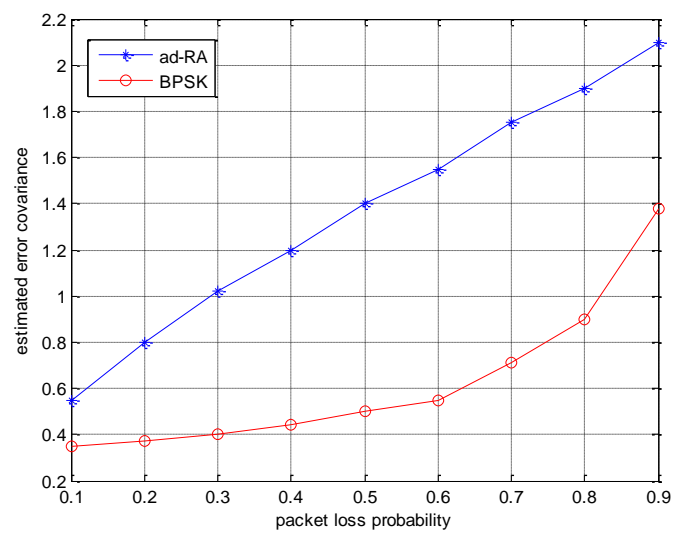

Figure 1. the relationship between estimation error covariance and packet loss probability

Figure 2 is the relationship between packet transmission energy and packet loss probability. From the simulation results, it can be found that with the increasing of packet loss probability, the data sent in the communication link is less and less, which leads directly to the decreasing of data transmission energy. However, this reduction in energy is at the expense of increased information loss, not the required optimal packet transmission energy. In the absence of network resource scheduling, the degraded communication quality caused by packet loss will make the packet transmission energy lose the optimal balance state and affect the network communication performance.

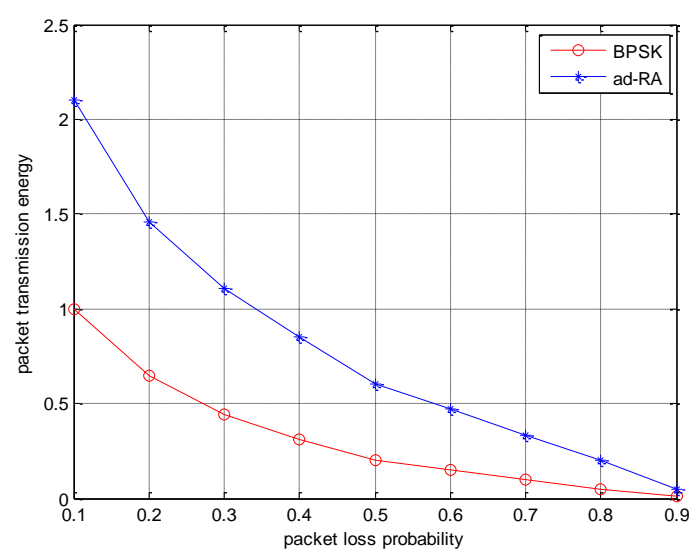

Figure 2. the relationship between packet transmission energy and packet loss probability

From the simulation results 1 and 2, we can find that the error covariance and the packet transmission energy are directly related to the packet loss probability. If there is no network resource scheduling, under the poor communication quality of the network, the estimated error covariance and packet transmission energy will become worse. It is necessary to consider the combined error of the estimated error covariance and packet transmission energy .

In the simulation of Figure 3, we set $T=1 \mathrm{~s}, \Gamma_{1}=20 \mathrm{~dB}, \Gamma_{2}=10 \mathrm{~dB}$. BPSK/QPSK and ad-RA, the three modulation methods use the same simulation parameter. We put them in the same environment simulation, and get their contrast effect. From the results 
of the simulation experiment, it can be seen that the objective function decreases with the increase of the bandwidth. When the bandwidth increases, the communication link has sufficient bandwidth available, then communication quality is getting better and better. From the simulation results, it can be seen that network optimization goals tend to be stable. In the bandwidth range, $15 \leq W_{B} \leq 30$, bandwidth resource scheduling is optimal. When the bandwidth resources increase, the quantization bit data will become larger, but the optimization target has no significant improvement. So that the quantization noise $n_{i, k}$ is negligible with respect to the observed noise $v_{i, k}$. Therefore, when the bandwidth is large enough, BPSK modulation is better, in this way the packet reception probability is greater than the QPSK mode packet reception probability.

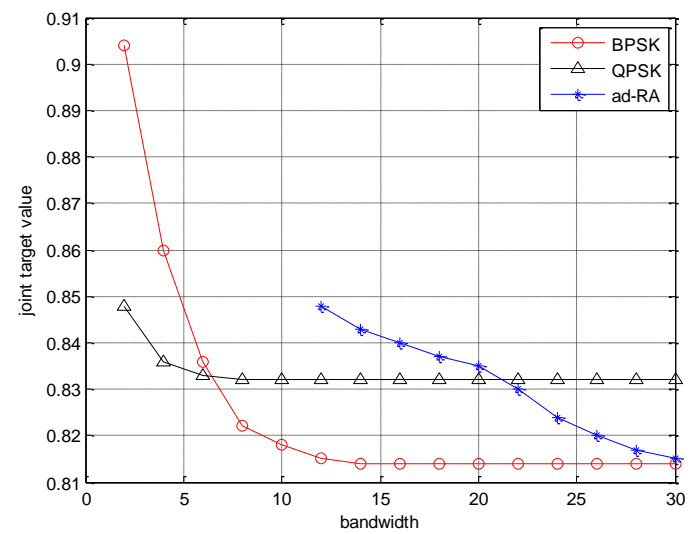

Figure 3. the relationship between joint optimization goal and network bandwidth

\section{Conclusion}

In this paper, for wireless communication link packet loss, under the simple BPSK modulation mode, it is possible to minimize the energy consumption of the network communication while minimizing the error covariance of the state, by optimizing the network resource scheduling, optimizing the network transmission bandwidth, bit data rate and scheduling of the transmission time. This kind of network resource scheduling method can effectively reduce the influence of information loss in the communication link on the state estimation and make up the shortcomings of packet loss in the wireless network.

\section{References}

1. Michael KintnerMeyer. Opportunities of Wireless Sensors and Controls for Building Operation[J]. Cell Biology International, Vol. 33, No. 9, 941-8,(2005).

2. Piran M J, Murthy G R, Babu G P. Vehicular Ad Hoc and Sensor Networks; Principles and Challenges[J]. International Journal of Ad Hoc Sensor \& Ubiquitous Computing, Vol. 2, No. 2, 3849,(2011).
3. Willig A, Matheus $\mathrm{K}$, Wolisz A. Wireless Technology in Industrial Networks[J]. Proceedings of the IEEE, Vol. 93, No. 6, 1130-1151,(2005).

4. Oh S, Schenato L, Chen P, et al. Tracking and Coordination of Multiple Agents Using Sensor Networks: System Design, Algorithms and Experiments[J]. Proceedings of the IEEE, Vol. 95, No. 1, 234-254,(2007).

5. Sinopoli B, Schenato L, Franceschetti $M$, et al. Kalman filtering with intermittent observations[J]. IEEE transactions on Automatic Control, Vol. 49, No. 9, 1453-1464,(2014).

6. Nair G N, Fagnani F, Zampieri S, et al. Feedback Control Under Data Rate Constraints: An Overview[J]. Proceedings of the IEEE,Vol. 95, No. 1,108 - 137,(2007).

7. Braslavsky J H, Middleton R H, Freudenberg J S. Feedback Stabilization Over Signal-to-Noise Ratio Constrained Channels[J]. IEEE Transactions on Automatic Control, Vol. 52, No. 8,13911403,(2007).

8. You K, Xie L. Mean square stabilizability of linear systems with limited feedback data rates and Markovian packet losses[C] Chinese Control Conference.IEEE, Vol. 22, No. 3, 4323-4328,(2010). 